\title{
Temperature inversion in long-range interacting systems
}

\author{
Tarcísio N. Teles, ${ }^{1,2, *}$ Shamik Gupta, ${ }^{3, \dagger}$ Pierfrancesco Di Cintio, ${ }^{3, \ddagger}$ and Lapo Casetti ${ }^{3,4, \S}$ \\ ${ }^{1}$ Instituto de Física, Universidade Federal do Rio Grande do Sul, Caixa Postal 15051, CEP 91501-970, Porto Alegre, RS, Brazil \\ ${ }^{2}$ Departamento de Ciências Exatas e Sociais Aplicadas, Universidade Federal de Ciências da Saúde de Porto Alegre, \\ CEP 90050-170, Porto Alegre, RS, Brazil \\ ${ }^{3}$ Dipartimento di Fisica e Astronomia and CSDC, Università di Firenze, and INFN, Sezione di Firenze, via G. Sansone 1, \\ I-50019 Sesto Fiorentino, Italy \\ ${ }^{4}$ INAF-Osservatorio Astrofisico di Arcetri, Largo E. Fermi 5, I-50125 Firenze, Italy
}

(Received 22 January 2015; revised manuscript received 29 May 2015; published 3 August 2015)

\begin{abstract}
Temperature inversions occur in nature, e.g., in the solar corona and in interstellar molecular clouds: Somewhat counterintuitively, denser parts of the system are colder than dilute ones. We propose a simple and appealing way to spontaneously generate temperature inversions in systems with long-range interactions, by preparing them in inhomogeneous thermal equilibrium states and then applying an impulsive perturbation. In similar situations, short-range systems would typically relax to another thermal equilibrium, with a uniform temperature profile. By contrast, in long-range systems, the interplay between wave-particle interaction and spatial inhomogeneity drives the system to nonequilibrium stationary states that generically exhibit temperature inversion. We demonstrate this mechanism in a simple mean-field model and in a two-dimensional self-gravitating system. Our work underlines the crucial role the range of interparticle interaction plays in determining the nature of steady states out of thermal equilibrium.
\end{abstract}

DOI: 10.1103/PhysRevE.92.020101

PACS number(s): 05.20.-y, 52.65.Ff, 96.60.P-, 98.38.Dq

Stationary states far from thermal equilibrium occur in nature. In some cases, e.g., in the solar corona and in interstellar molecular clouds, such states exhibit temperature inversion: Denser parts of the system are colder than dilute ones. This work is motivated by an attempt to explain how such a counterintuitive effect may spontaneously arise in nonequilibrium states, unveiling its minimal ingredients and the underlying physical mechanism. To this end, we start with asking a simple yet physically relevant question: What happens if an isolated macroscopic system in thermal equilibrium is momentarily disturbed, e.g., by an impulsive force or a "kick"? If the interactions among the system constituents are short ranged, collisions redistribute the kick-injected energy among the particles, yielding a fast relaxation to a new equilibrium, with a Maxwellian velocity distribution and a uniform temperature across the system.

Is the scenario the same if instead the interactions are long ranged [1]? For long-range systems, collisional effects act over a characteristic time $\tau_{\text {coll }}$ that, unlike short-range systems, scales with the system size $N$, diverging as $N \rightarrow \infty$ [2]. As a result, a macroscopic system with long-range interactions starting from generic initial conditions will attain thermal equilibrium only after extremely long times, often exceeding typical observation times. Examples of long-range systems are self-gravitating systems, for which, e.g., $\tau_{\text {coll }} \simeq 10^{10}$ years for globular clusters and orders of magnitude larger than the age of the universe for galaxies [3,4]. The collisionless evolution of long-range interacting systems for times shorter than $\tau_{\text {coll }}$ is governed by the Vlasov (or collisionless Boltzmann) equation [2]. When kicked out of thermal equilibrium, a long-range

\footnotetext{
*tarcisio.nteles@gmail.com

${ }^{\dagger}$ shamikg1@gmail.com

${ }^{\ddagger}$ pierfrancesco.dicintio@unifi.it

§lapo.casetti@unifi.it
}

interacting system relaxes to a Vlasov-stationary state, and thermal equilibrium is just one of infinitely many possible states. Predicting which Vlasov state follows a given initial condition is an extremely difficult problem [5], first addressed in Ref. [6] and still unsolved, despite some remarkable achievements for particular systems and special initial conditions (see Refs. [7,8]).

Let us then ask a simpler question: How different from an equilibrium state is the stationary state the system relaxes to after the kick? Are there ways to characterize it, e.g., by unveiling some of its general features? The answer is yes; in this Rapid Communication, we argue that, provided it started from a spatially inhomogeneous equilibrium state [9], the system after the kick relaxes to a state with a nonuniform temperature profile. In short-range systems, by contrast, a nonuniform temperature profile may only occur when the system is actively maintained out of equilibrium, e.g., by a boundary-imposed temperature gradient, to counteract collisional effects. Remarkably, in a long-range system, the relaxed state after the kick generically exhibits temperature inversion, as we will explicitly show.

As recalled above, temperature inversions are observed [10] in interstellar molecular clouds [11-13] and especially in the solar corona, where temperatures around $10^{6} \mathrm{~K}$ that are three orders of magnitude larger than the temperature of the photosphere are attained [14]. Despite recent advances [15], the mechanism of coronal heating is not completely understood and remains one of the most important open problems in astrophysics [16]. Most attempts to explain such a phenomenon involve mechanisms that actively inject energy [17] into the less dense regions of the system. A different possibility, suggested by Scudder [18-20], is referred to as velocity filtration; see also Ref. [21] and the Supplemental Material (SM) [22]. Consider a system of particles acted upon by an external field whose potential energy increases with height above a base level. Only particles with a sufficiently 
large kinetic energy can climb the potential well and reach a given height. If the velocity distribution at the base is Maxwellian, it remains as such with the system maintaining the same temperature at all heights in the stationary state. Instead, if the distribution is suprathermal, i.e., with tails fatter than Maxwellian, the temperature in the stationary state increases with height, with a concomitant decrease of the density. It is argued $[16,23,24]$ that velocity filtration might not be the (only) mechanism behind coronal heating, but nevertheless provides a simple and appealing explanation of how a counterintuitive temperature inversion occurs without steady energy injection in less dense parts of the system.

Scudder's original model neglects interparticle interactions and requires an "active" ingredient, i.e., an out-of-equilibrium suprathermal velocity distribution imposed as a boundary condition. It was recently shown [21] that temperature inversion occurs also in strongly interacting systems, provided the interactions are long ranged, when a velocity distribution with suprathermal tails is given just as the initial condition of the dynamical evolution. Although much weaker than a nonthermal boundary condition, the latter is still an ad hoc requirement. However, it is not necessary at all: In this Rapid Communication, we demonstrate that temperature inversion emerges spontaneously in the stationary state reached after a long-range system is brought out of equilibrium by a perturbation acting for a very short time, and there is no need for a suprathermal initial distribution. Our work thus suggests that temperature inversions observed in astrophysical systems may be examples of a more general phenomenon, whose roots are in the long-range nature of the interparticle interactions.

Let us consider the very general setting of a system of $N$ interacting particles of mass $m$ in $d$ dimensions with the Hamiltonian

$$
\mathcal{H}=\sum_{i=1}^{N} \frac{p_{i}^{2}}{2 m}+\sum_{i=1}^{N} \sum_{j>i}^{N} V\left(\left|\mathbf{r}_{i}-\mathbf{r}_{j}\right|\right),
$$

where $p_{i}=\left|\mathbf{p}_{i}\right|$ are the momenta conjugated to the positions $\mathbf{r}_{i}$, the potential energy is long ranged, $V(r) \propto r^{-\alpha}, 0 \leqslant \alpha \leqslant$ $d$ as $r \rightarrow \infty$. For times $t \ll \tau_{\text {coll }}$, the dynamics is described by the single-particle phase space distribution $f(\mathbf{r}, \mathbf{p}, t)$ obeying the Vlasov equation

$$
\frac{\partial f}{\partial t}+\mathbf{p} \cdot \nabla_{\mathbf{r}} f-\nabla_{\mathbf{r}} u[f] \cdot \nabla_{\mathbf{p}} f=0,
$$

where

$$
u[f] \equiv \int d \mathbf{r} d \mathbf{p} f(\mathbf{r}, \mathbf{p}, t) V(r)
$$

is the mean-field potential energy. An initial condition $f_{0}=$ $f(\mathbf{r}, \mathbf{p}, 0)$ chosen to be a stationary solution of Eq. (2) does not evolve in time. Otherwise, if the initial distribution is not a stationary solution of (2), after a short transient (often referred to as "violent relaxation," after Lynden-Bell [6]), the system settles into a stable stationary solution of Eq. (2) called a quasistationary state (QSS), in which the system remains trapped until, at $t \simeq \tau_{\text {coll }}$, collisional effects neglected in Eq. (2) drive the system towards thermal equilibrium [2]. To construct a first representative example to demonstrate our claims, we assume periodic coordinates so that boundary effects may be neglected [25] and expand the interparticle potential (that by definition is even) in a cosine Fourier series. We then set $d=1$ and retain just the first Fourier term. The resulting model, the so-called Hamiltonian mean-field (HMF) model, describes a system of globally interacting point particles moving on a circle with Hamiltonian [26]

$$
\mathcal{H}_{\mathrm{HMF}}=\sum_{i=1}^{N} \frac{p_{i}^{2}}{2}+\frac{1}{N} \sum_{i=1}^{N} \sum_{j>i}^{N}\left[1-\cos \left(\vartheta_{i}-\vartheta_{j}\right)\right],
$$

where $\vartheta_{i} \in(-\pi, \pi]$ is the angular coordinate of the $i$ th particle on the circle, while $p_{i}$ is the conjugated momentum; we have further assumed $m=1$ and the interaction to be attractive, fixing the energy scale to unity, and scaled it by $1 / N$ to make it extensive (Kac prescription [2]). The Hamiltonian (4) is invariant under the $O(2)$ symmetry group. In thermal equilibrium, for energy density $\varepsilon=E / N$ smaller than $\varepsilon_{c}=$ $3 / 4$, the symmetry is spontaneously broken to result in a clustered state. The order parameter of clustering is the average magnetization [27] $\left(m_{x}, m_{y}\right) \equiv \frac{1}{N}\left(\sum_{i=1}^{N} \cos \vartheta_{i}, \sum_{i=1}^{N} \sin \vartheta_{i}\right)$. The HMF model is a simple system which, besides serving as a framework to study statics and dynamics of longrange systems, actually models physical systems such as gravitational sheet models and free-electron lasers [2].

In order to study what happens when we "kick" the HMF system out of equilibrium, we studied its dynamical evolution via molecular dynamics (MD) simulations, performed by integrating [28] the equations of motion derived from the Hamiltonian (4). We prepared the system in an equilibrium state with $m_{x}=m_{0}$ and $m_{y}=0$, and with a Maxwellian velocity distribution corresponding to the equilibrium temperature. We let the system evolve until $t=t_{0}>0$, and then kicked it out of equilibrium by applying during a short time $\tau$ an external magnetic field $h$ along the $x$ direction; thus, for $t_{0}<t<t_{0}+\tau$, the Hamiltonian (4) is augmented by $\mathcal{H}_{h}=-h \sum_{i=1}^{N} \cos \vartheta_{i}$. Here we present results for $t_{0}=100$, $\tau=1, h=10$, and $m_{0}=0.521$, corresponding to an initial equilibrium temperature $T=0.4244$. We considered up to $N=10^{7}$ particles. After the kick the magnetization starts oscillating, and after a transient the oscillations damp down and a new stationary value $m^{*}<m_{0}$ of the magnetization is reached. A typical time evolution of the magnetization is shown in Fig. 1.

The fact that $m^{*}<m_{0}$ is not surprising because the system gains energy during the interaction with the external field $h$, resulting in an energy density $\varepsilon^{*}>\varepsilon_{c}$, and if the system would relax to a new equilibrium, the latter would have a magnetization $m_{\mathrm{eq}}=0$. The stationary state reached after the damping of the oscillations is a QSS, very far from the homogeneous equilibrium at $\varepsilon^{*}$. The nonequilibrium character of this state is further shown by the fact that the temperature profile

$$
T(\vartheta) \equiv \frac{\int_{-\infty}^{\infty} d p p^{2} f(\vartheta, p)}{\int_{-\infty}^{\infty} d p f(\vartheta, p)}
$$

is nonuniform, and there is temperature inversion, as shown in Fig. 2, where $T(\vartheta)$ is plotted together with the density profile

$$
n(\vartheta) \equiv \int_{-\infty}^{\infty} d p f(\vartheta, p) .
$$




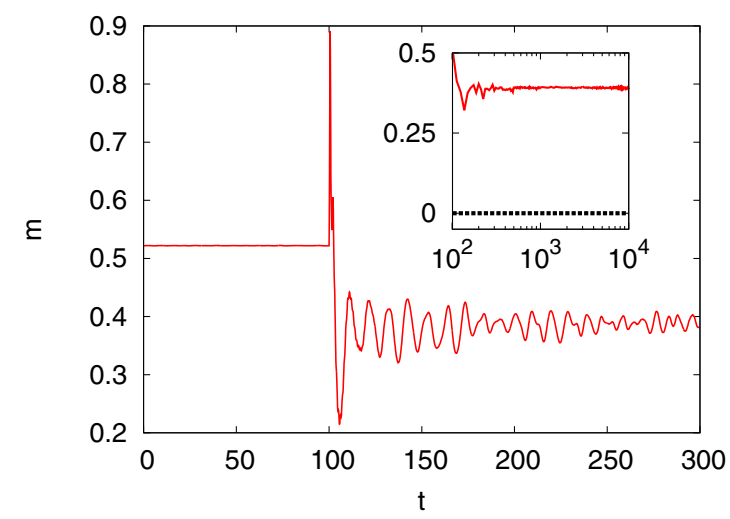

FIG. 1. (Color online) HMF: Time evolution of the magnetization $m$ with $N=10^{7}$ (solid red line). Inset: $m(t)$ compared to the equilibrium value $m_{\mathrm{eq}}=0$ (dotted black line) for longer times.

The temperature profile indeed remains essentially the same for the whole lifetime of the QSS, as we checked by measuring an integrated distance $\xi$ between the actual temperature profile and the constant equilibrium one, $T_{\text {eq }}$, at the same energy, as follows:

$$
\xi(t) \equiv \int_{-\pi}^{\pi}\left|T(\vartheta, t)-T_{\mathrm{eq}}\right| d \vartheta .
$$

In Fig. 3, $\xi(t)$ is plotted for systems with different values of $N$ kicked with the same $h=10$ at $t_{0}=100$ for a duration $\tau=1$. After the kick, $\xi(t)$ oscillates and then reaches a plateau whose duration grows with $N$, as expected for a QSS. The inset of Fig. 3 shows that if times are scaled by $N$, the curves reach zero at the same time, consistently with the lifetime of an inhomogeneous QSS being proportional to $N$ [29].

We performed simulations starting with equilibrium states corresponding to different values of $m_{0}$ and applying perturbations of different strengths $h$ and duration $\tau$, and the system almost always ended up in a QSS, showing at least a partial temperature inversion over some interval of the values of $\vartheta$, if not the whole; we also considered another model where also the second Fourier mode is retained, again obtaining the same qualitative behavior (see Supplemental Material [22]).

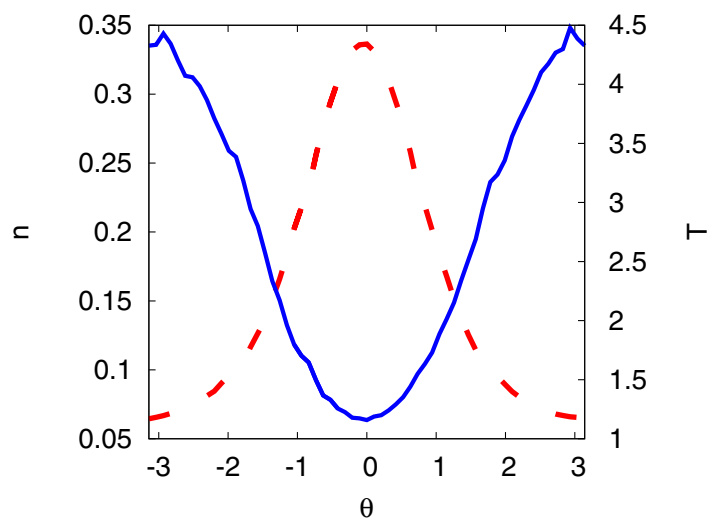

FIG. 2. (Color online) HMF: Temperature profile $T(\vartheta)$ (blue solid line) and density profile $n(\vartheta)$ (red dashed line), measured in the QSS at $t=10^{4}$.

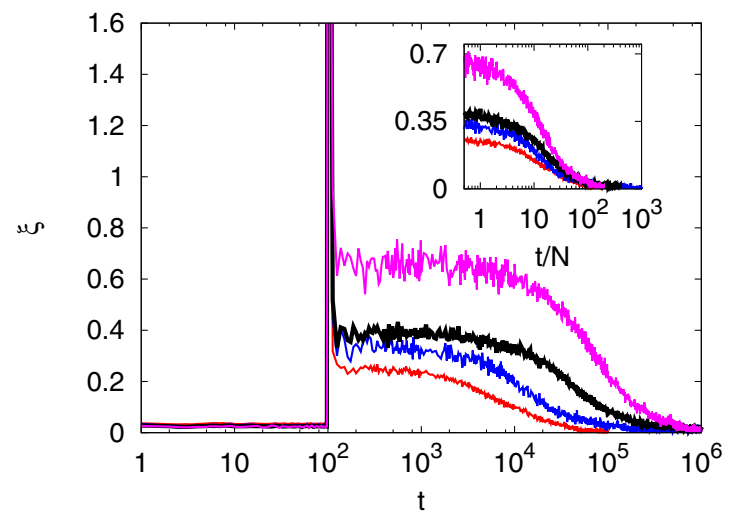

FIG. 3. (Color online) HMF: Time evolution of the distance from equilibrium temperature $\xi$, Eq. (7), with $N$ increasing from bottom to top: $N=5 \times 10^{2}$ (red), $N=10^{3}$ (blue), $N=2.5 \times 10^{3}$ (black), $N=5 \times 10^{3}$ (purple). Each curve is the average over $n_{r}$ realizations with $n_{r}$ ranging from 20 for $N=5 \times 10^{3}$ to $10^{3}$ for $N=5 \times 10^{2}$. Inset: $\xi$ as a function of $t / N$.

Preparing the system in equilibrium and then bringing it out of equilibrium by means of a perturbation acting for a short time mimics processes that may actually happen in nature, e.g., a transient density perturbation in a self-gravitating fluid. Hence, as a second example, we considered a two-dimensional self-gravitating system (2DSGS), that is, a system of $N$ particles of mass $m$ moving in a plane with the Hamiltonian

$$
\mathcal{H}_{\mathrm{G}}=\sum_{i=1}^{N} \frac{\left|\mathbf{p}_{i}\right|^{2}}{2 m}+G m^{2} \sum_{i, j>i}^{N} \ln \left[\frac{\sqrt{\left(\mathbf{r}_{i}-\mathbf{r}_{j}\right)^{2}+r_{0}^{2}}}{s}\right],
$$

where $\mathbf{r}_{i}=\left(x_{i}, y_{i}\right), s$ is a length scale, $\mathbf{p}_{i}=\left(\dot{x}_{i}, \dot{y}_{i}\right), r_{0}$ is a small-scale cutoff, and $G$ is the gravitational constant. Such a system can be seen as a simple model of filamentary interstellar clouds [30]. We performed MD simulations considering $N=3 \times 10^{4}$ particles initially in a thermal equilibrium state, whose radial density profile is known [31] (see Supplemental Material [22]), and we kicked them out of equilibrium by instantaneously adding to all their radial velocities the same amount $\delta v_{r}=\sigma_{r} / 2$, where $\sigma_{r}$ is the radial velocity dispersion. As in the HMF case, after the kick the system develops macroscopic oscillations that damp out after a time of order $\tau_{\text {dyn }}=r_{*}^{0} \sqrt{2 / G M}$, where $M$ is the total mass and $r_{*}^{0}=r_{*}(t=$ 0 ) is the (initial) half-mass radius, and eventually sets in a QSS. The latter exhibits temperature inversion up to $r \gtrsim r_{*}(t)$, as shown in Fig. 4. The radial profiles $n(r)$ and $T(r)$ are obtained by averaging $T(\mathbf{r})$ and $n(\mathbf{r})$, defined by replacing $(\vartheta, p)$ with $(\mathbf{r}, \mathbf{p})$ in Eqs. (5) and (6), over the polar angle.

We have thus shown that temperature inversion arises in two different long-range-interacting systems when kicked out of equilibrium. Moreover, also if we start from a state with a Maxwellian velocity distribution but a spatial distribution different from the equilibrium one, macroscopic oscillations develop, and after the damping of the oscillations, the systems end up in a QSS, typically exhibiting at least a partial temperature inversion (see Supplemental Material [22]). Therefore, this phenomenon does not depend on the details of the way 


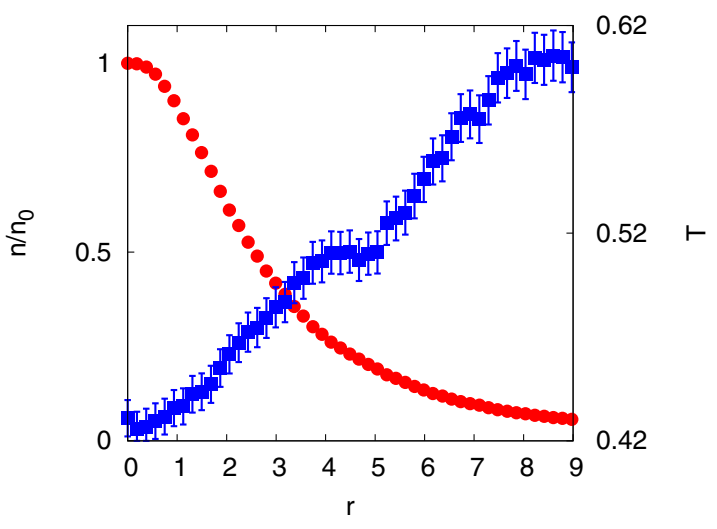

FIG. 4. (Color online) 2DSGS: Temperature profile $T(r)$ (blue squares) and density profile $n(r) / n_{0}$ (red circles) measured in the QSS at $t=90 \tau_{\text {dyn }}$ while starting from a thermal state with uniform temperature $T_{0}=0.5$, in natural units such that $r_{*}(t) \simeq 7$ and $r_{0}=10^{-3}$.

the initial state is prepared, provided it is inhomogeneous, and that a collective oscillation develops when it is brought out of equilibrium.

A very general mechanism may then be responsible for this phenomenology. In the following, we argue that indeed a simple and general explanation can be found. As stated above, before collisional effects set in, the dynamics of a long-range interacting system is described by the Vlasov equation (2). When a state described by a stationary solution of the Vlasov equation is perturbed, a phenomenon called Landau damping [32] occurs, which is a kind of waveparticle interaction responsible for the collisionless damping of oscillations induced by the external perturbation. The theory of Landau damping is well developed, and, in the case of small perturbations of homogeneous states, has been recently put on rigorous grounds [33]. The phenomenon occurs also when the perturbed state is inhomogeneous [34,35]. The basic physical mechanism is the following: The perturbation creates a wave in the system, as witnessed by the onset of collective oscillations. Consider for simplicity a monochromatic wave. Particles that have a velocity $v$ close to the phase velocity $v_{\text {ph }}$ of the wave will interact strongly with the perturbation, while those with $v \ll v_{\text {ph }}$ or $v \gg v_{\text {ph }}$ will be essentially unaffected. Particles with velocities slightly less than $v_{\mathrm{ph}}$ will acquire kinetic energy, while those with velocities slightly larger than $v_{\mathrm{ph}}$ will lose kinetic energy. Since for a typical $f(v)$ one has $d f / d v<0$ (for positive $v$, and the opposite for negative $v$ ), there are more particles that gain energy than those that lose energy, so that the wave in effect loses energy, and the perturbation is damped. After the interaction with the perturbation, a Maxwellian $f(v)$ is modified close to $v_{\text {ph }}$ (and if the wave is stationary or travels in both directions, as in our case, also close to $-v_{\mathrm{ph}}$ ), where a "shoulder" is formed, and $\left\langle v^{2}\right\rangle$ is increased. If, prior to perturbation, the state is spatially homogeneous, this happens in the same way throughout the system, and the initially coherent energy of the wave goes into uniformly heating the system. But in our case the state is not homogeneous prior to the perturbation: It is clustered, and the "shoulder" in $f(v)$ created by Landau damping is nothing but a suprathermal tail, so that now velocity filtration acts and produces temperature inversion-fast particles climb the potential well higher than slower ones, and suprathermal tails grow when density decreases. Indeed, as shown in Ref. [21], velocity filtration always works in systems described by the Vlasov equation (2); there, the suprathermal tails of $f(v)$ were fed by the initial conditions, while here it is the perturbation that creates them, via Landau damping. It is interesting to note that standing radio-frequency waves are used to heat laboratory plasmas by creating a shoulder in the velocity distribution [36]; in that case, one needs to sustain the wave from outside, while in our example it is velocity filtration that amplifies the effect.

The above physical picture is idealized and one should take into account the coupling of the various modes of the perturbation in the inhomogeneous case [34,35]. This notwithstanding, the main point is that the perturbation does not interact in the same way with all the particles, but preferentially gives energy to particles with already rather large velocities. This results in a suprathermal velocity distribution, as shown in Fig. 5 (left panel) for the HMF model. Soon after the kick a high- $p$ tail shows up, which is built up from peaks at different values of $p$ corresponding to different positions. The evolution of these peaks results in oscillations in $f(p)$ for
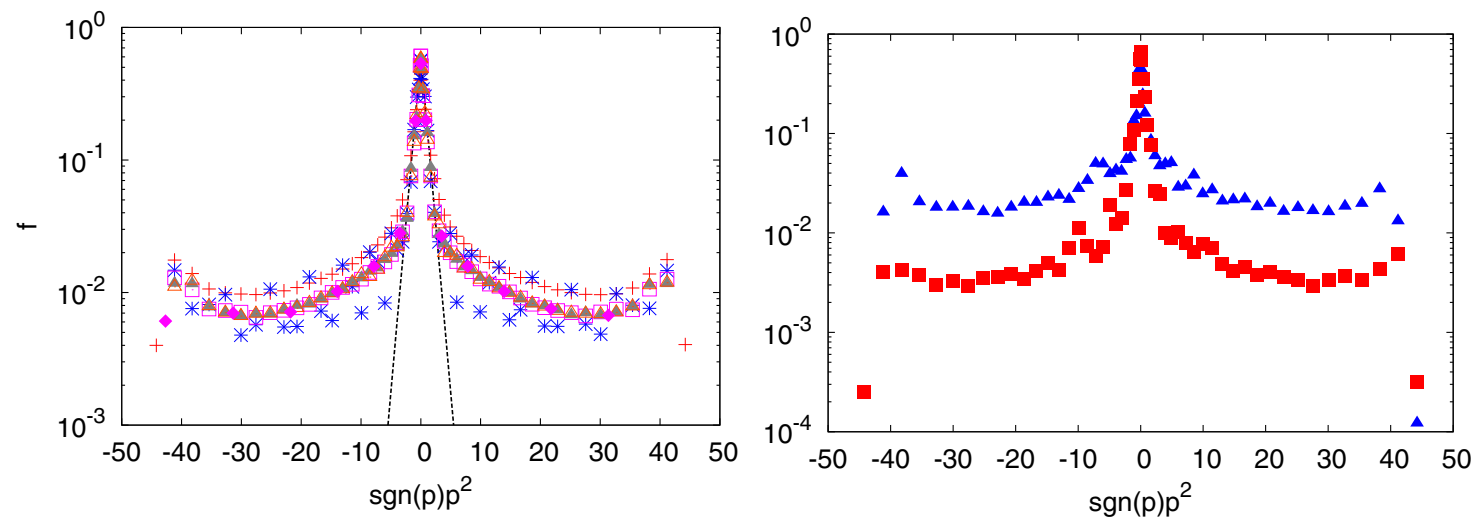

FIG. 5. (Color online) HMF. Left: Momentum distribution $f(p)$ at $t=0$ (black dashed line), $t=101$ (red plus signs), $t=110$ (blue asterisks), $t=150$ (magenta open squares), $t=200$ (gray solid triangles), $t=10^{3}$ (orange open triangles), and $t=10^{4}$ (magenta solid rhombi). Right: Distribution function $f(\vartheta, p)$ measured in the QSS as in Fig. 2, at $\vartheta=0$ (red squares) and $\vartheta=\pi$ (blue triangles). The distribution functions are plotted against $p^{2} \operatorname{sgn}(p)$ to better show the difference with respect to the initial Maxwellian. 
$t \lesssim 150$, then the oscillations damp out, and due to the absence of an efficient mechanism able to evenly redistribute this excess energy among all the particles, the velocity distribution stays nonthermal and essentially the same for times $t<\tau_{\text {coll }}$, allowing velocity filtration to produce temperature inversion. The distribution function of the HMF model in the QSS is plotted for two different values of $\vartheta$ in Fig. 5 (right panel), and the growth of the suprathermal tails in the less dense parts of the system is well apparent. We found similar results for the distribution functions also in the 2DSGS case (see Supplemental Material [22]). For the HMF, perturbations of the form $-h \sum_{i=1}^{N} \cos \left(k \vartheta_{i}\right)$ with $k \neq 1$ also yield preferential absorption of energy around values of $v$ that are different from those in the $k=1$ case, coherently with the above picture (see Supplemental Material [22]).
We have shown that nonequilibrium stationary states with temperature inversions are the typical outcome of a perturbation acting for a short time on a clustered equilibrium state of a long-range interacting system. This rather surprising result can be explained in terms of Landau damping and velocity filtration, suggesting that temperature inversions may occur whenever the dynamics is collisionless up to the relevant time scales. This mechanism may be actually relevant to understand temperature inversions observed in nature.

We thank the Galileo Galilei Institute for Theoretical Physics, Florence, Italy for the hospitality and the INFN (Italy) and the CNPq (Brazil) for partial support during the completion of this work. L.C. thanks D. Galli for very useful discussions. S.G. and T.N.T. contributed equally to this work.
[1] Interactions are called long range when they decay asymptotically with the interparticle distance $r$ as $r^{-\alpha}$ with $0 \leqslant \alpha \leqslant d$ in $d$ dimensions [2].

[2] A. Campa, T. Dauxois, D. Fanelli, and S. Ruffo, Physics of LongRange Interacting Systems (Oxford University Press, Oxford, UK, 2014).

[3] A. R. Choudouri, Astrophysics for Physicists (Cambridge University Press, Cambridge, UK, 2010).

[4] J. Binney and S. Tremaine, Galactic Dynamics, 2nd ed. (Princeton University Press, Princeton, NJ, 2008).

[5] Also, the related issue of stability of the Vlasov stationary states is resolved only in particular cases [2].

[6] D. Lynden-Bell, Mon. Not. R. Astron. Soc. 136, 101 (1967).

[7] Y. Levin, R. Pakter, F. B. Rizzato, T. N. Teles, and F. P. C. Benetti, Phys. Rep. 535, 1 (2014).

[8] F. P. C. Benetti, A. C. Ribeiro-Teixeira, R. Pakter, and Y. Levin, Phys. Rev. Lett. 113, 100602 (2014).

[9] At variance with short-range systems, equilibrium states of long-range systems can be inhomogeneous, typically when the interactions are attractive.

[10] Temperature inversions have been observed also in other systems such as the hot gas of "cooling-core" galaxy clusters [37] and the Io plasma torus around Jupiter [38].

[11] P. C. Myers and G. A. Fuller, Astrophys. J. 396, 631 (1992).

[12] A. A. Goodman et al., Astrophys. J. 504, 223 (1998).

[13] J. E. Pineda et al., Astrophys. J. Lett. 712, L116 (2010).

[14] L. Golub and J. M. Pasachoff, The Solar Corona, 2nd ed. (Cambridge University Press, Cambridge, UK, 2009).

[15] M. J. Aschwanden, Physics of the Solar Corona. An Introduction with Problems and Solutions, 2nd ed. (Springer, New York, 2005).

[16] J. A. Klimchuk, Sol. Phys. 234, 41 (2006).

[17] By means of, e.g., magnetic reconnection or Alfvén waves [16].
[18] J. D. Scudder, Astrophys. J. 398, 299 (1992).

[19] J. D. Scudder, Astrophys. J. 398, 319 (1992).

[20] J. D. Scudder, Astrophys. J. 427, 446 (1994).

[21] L. Casetti and S. Gupta, Eur. Phys. J. B 87, 91 (2014).

[22] See Supplemental Material at http://link.aps.org/supplemental/ 10.1103/PhysRevE.92.020101 for more details and results on numerical simulations and for a tutorial discussion of velocity filtration.

[23] S. W. Anderson, Astrophys. J. 437, 860 (1994).

[24] S. Landi and F. G. E. Pantellini, Astron. Astrophys. 372, 686 (2001).

[25] At variance with Scudder's model, we do not need any special boundary condition.

[26] M. Antoni and S. Ruffo, Phys. Rev. E 52, 2361 (1995).

[27] The HMF may also be seen as a system of mean-field $X Y$ spins.

[28] We used a fourth-order symplectic algorithm with time step $\delta t=0.1$, keeping relative energy fluctuations below $10^{-8}$.

[29] P. de Buyl, D. Mukamel, and S. Ruffo, Phys. Rev. E 84, 061151 (2011).

[30] C. Toci and D. Galli, Mon. Not. R. Astron. Soc. 446, 2110 (2015).

[31] J. Ostriker, Astrophys. J. 140, 1056 (1964).

[32] A. R. Choudouri, The Physics of Fluids and Plasmas (Cambridge University Press, Cambridge, UK, 1998).

[33] C. Mohout and C. Villani, Acta Math. 207, 29 (2011).

[34] J. Barré, A. Olivetti, and Y. Y. Yamaguchi, J. Stat. Mech.: Theory Exp. (2010) P08002.

[35] J. Barré, A. Olivetti, and Y. Y. Yamaguchi, J. Phys. A: Math. Theor. 44, 405502 (2011).

[36] K. J. Bowers and C. K. Birdsall, Phys. Plasmas 9, 2405 (2002).

[37] M. W. Wise, B. R. McNamara, and S. S. Murray, Astrophys. J. 601, 184 (2004).

[38] N. Meyer-Vernet, M. Moncuquet, and S. Hoang, Icarus 116, 202 (1995). 